\title{
A REFINED POLAR DECOMPOSITION FOR $J$-UNITARY OPERATORS
}

\author{
SERGEY M. ZAGORODNYUK
}

\begin{abstract}
In this paper, we will characterize the components of the polar decomposition for an arbitrary $J$-unitary operator in a Hilbert space (where $J$ is a conjugation). This characterization has a quite different structure from that of symmetric and complex skew-symmetric operators. It is also shown that for a $J$-imaginary closed symmetric operator in a Hilbert space there exists a $J$-imaginary self-adjoint extension in a possibly larger Hilbert space (a linear operator $A$ in a Hilbert space $H$ is said to be $J$-imaginary if $f \in D(A)$ implies $J f \in D(A)$ and $A J f=-J A f$, where $J$ is a conjugation on $H$.) All Hilbert spaces in this paper are assumed to be separable.
\end{abstract}

\section{INTRODUCTION}

In recent years, $J$-symmetric, $J$-skew-symmetric and $J$-unitary operators have attracted the attention of researchers, see, e.g., [4]-[6] and references therein. Recall that a conjugation $J$ in a Hilbert space $H$ is an antilinear operator on $H$ such that $J^{2} x=x, x \in H$, and

$$
(J x, J y)_{H}=(y, x)_{H}, \quad x, y \in H .
$$

The conjugation $J$ generates the following bilinear form:

$$
[x, y]_{J}:=(x, J y)_{H}, \quad x, y \in H .
$$

A linear operator $A$ in $H$ is said to be $J$-symmetric ( $J$-skew-symmetric) if

$$
[A x, y]_{J}=[x, A y]_{J}, \quad x, y \in D(A),
$$

or, respectively,

$$
[A x, y]_{J}=-[x, A y]_{J}, \quad x, y \in D(A) .
$$

2010 Mathematics Subject Classification. Primary 47A05: Secondary: 47A20, 47B15. Key words and phrases. Polar decomposition, conjugation, J-unitary operator, symmetric operator.

Copyright (c) 2015 by ANUBiH. 
A linear operator $A$ in $H$ is said to be $J$-isometric if

$$
[A x, A y]_{J}=[x, y]_{J}, \quad x, y \in D(A) .
$$

A linear operator $A$ in $H$ is called $J$-self-adjoint ( $J$-skew-self-adjoint, or $J$-unitary) if

$$
A=J A^{*} J
$$

or

$$
A=-J A^{*} J
$$

or

$$
A^{-1}=J A^{*} J
$$

respectively.

A refined polar decomposition for complex symmetric operators was obtained by Garcia and Putinar in [3]. Using the technique of Garcia and Putinar, an analog for complex skew-symmetric operators was obtained by $\mathrm{Li}$ and Zhou in [6, Lemma 2.3]. In this paper, we shall characterize the components of the polar decomposition for an arbitrary $J$-unitary operator. This characterization has a very different structure from that of the above mentioned decompositions. For the case of a bounded $J$-unitary operator, a similar decomposition was obtained in [9, Theorem 3.2]. However, in the unbounded case we can not use arguments from [9].

A linear operator $A$ in a Hilbert space $H$ is said to be $J$-imaginary $(J$ real) if $f \in D(A)$ implies $J f \in D(A)$ and $A J f=-J A f$ (respectively $A J f=J A f)$, where $J$ is a conjugation on $H$, see [8], [7]. We shall answer a question concerning the existence of $J$-imaginary self-adjoint extensions of $J$-imaginary symmetric operators. This subject is similar to the study of $J$ real self-adjoint extensions of $J$-real symmetric operators, see [8]. However, we can not state that a $J$-imaginary symmetric operator has equal defect numbers. Nevertheless, it is shown that a $J$-imaginary self-adjoint extension of a $J$-imaginary symmetric operator exists in a possibly larger Hilbert space.

Notations. As usual, we denote by $\mathbb{R}, \mathbb{C}, \mathbb{N}, \mathbb{Z}, \mathbb{Z}_{+}$, the sets of real numbers, complex numbers, positive integers, integers and non-negative integers, respectively; $\mathbb{R}_{e}=\mathbb{C} \backslash \mathbb{R}$. By $I_{d}$ we denote the unit matrix of order $d ; d \in \mathbb{N}$. By $\mathfrak{B}(S)$ we mean a set of all Borel subsets of $S \subseteq \mathbb{C}$. If $\mathrm{H}$ is a Hilbert space then $(\cdot, \cdot)_{H}$ and $\|\cdot\|_{H}$ mean the scalar product and the norm in $H$, respectively. Indices may be omitted in obvious cases. For a linear operator $A$ in $H$, we denote by $D(A)$ its domain, by $R(A)$ its range, and $A^{*}$ means the adjoint operator if it exists. If $A$ is invertible then $A^{-1}$ denotes its inverse. $\bar{A}$ means the closure of the operator, if the operator is closable. If $A=A^{*}$, then $\mathcal{R}_{z}(A):=\left(A-z E_{H}\right)^{-1}, z \in \mathbb{R}_{e}$. For a set $M \subseteq H$ we denote by $\bar{M}$ the closure of $M$ in the norm of $H$. By $E_{H}$ we denote the identity operator 
in $H$, i.e. $E_{H} x=x, x \in H$. In obvious cases we may omit the index $H$. All Hilbert spaces in this paper are assumed to be separable.

\section{Properties of $J$-Unitary operators}

The following proposition accumulates some basic properties of $J$-unitary operators.

Proposition 1. Let $J$ be a conjugation on a Hilbert space $H$, and $A$ be a $J$-unitary operator in $H$. Then the following statements are true:

(i) A is closed;

(ii) $A^{-1}$ is J-unitary;

(iii) $A^{*}$ is J-unitary;

(iv) $A^{*} A$ is J-unitary;

(v) If $A$ is bounded, then $D(A)=R(A)=H$.

Proof. Let $A$ be a $J$-unitary operator in a Hilbert space $H$. By $[9$, Proposition 2.8] we may write: $A^{-1}=J A^{*} J=(J A J)^{*}$, and therefore $A^{-1}$ and $A$ are closed. Moreover, by [9, Proposition 2.10] we get

$$
\left(A^{-1}\right)^{-1}=\left(J A^{*} J\right)^{-1}=J\left(A^{*}\right)^{-1} J=J\left(A^{-1}\right)^{*} J,
$$

and therefore $A^{-1}$ is $J$-unitary. Since

$$
\left(A^{*}\right)^{-1}=\left(A^{-1}\right)^{*}=J A J
$$

then $A^{*}$ is $J$-unitary. Set $G=A^{*} A$. The operator $G$ is non-negative and we may write:

$$
J G^{*} J=J A^{*} A J=J A^{*} J J A J=A^{-1}\left(A^{*}\right)^{-1}=\left(A^{*} A\right)^{-1} .
$$

Thus, $G$ is $J$-unitary.

If $A$ is bounded, then $A^{-1}=J A^{*} J$ is bounded, and the closeness of $A$ and $A^{-1}$ implies (v).

Now we can obtain a refined polar decomposition for a $J$-unitary operator.

Theorem 2.1. Let $J$ be a conjugation on a Hilbert space $H$. Then the following assertions hold:

1) If $A$ is a J-unitary operator in $H$ then

$$
A=U B,
$$

where $U$ is a unitary $J$-real operator, and $B$ is a non-negative selfadjoint $J$-unitary operator;

2) If an operator $A$ in $H$ admits a representation (8) with a unitary $J$ real operator $U$, and a non-negative self-adjoint $J$-unitary operator $B$, then $A$ is J-unitary. 
Proof. Let $A$ be a $J$-unitary operator in a Hilbert space $H$. Set $G=A^{*} A$, and let $E_{G}(\delta), \delta \in \mathfrak{B}(\mathbb{R})$ be the spectral measure of $G$. By Proposition 1 we conclude that $G$ is $J$-unitary. Let us check that $E(\delta):=J E_{G}(\delta) J, \delta \in \mathfrak{B}(\mathbb{R})$ is the spectral measure of $G^{-1}$. In fact, $E(\delta)$ satisfies conditions $E^{2}=E$, $E^{*}=E$, therefore $E(\delta)$ is a projection operator. The strong $\sigma$-additivity of $E$ follows from the strong $\sigma$-additivity of $E_{G}$ and the continuity of $J$. Moreover, $E(\mathbb{R})=J E_{G}(\mathbb{R}) J=E_{H}$. Thus, $E$ is a spectral measure. Denote by $T$ the self-adjoint operator in $H$ corresponding to $E$. Observe that

$$
\begin{aligned}
\mathcal{R}_{z}\left(G^{-1}\right)=\left(G^{-1}-z E_{H}\right)^{-1} & =\left(J G^{*} J-J \bar{z} J\right)^{-1} \\
& =J\left(G^{*}-\bar{z} E_{H}\right)^{-1} J=J \mathcal{R}_{z}^{*}(G) J, \quad z \in \mathbb{R}_{e} .
\end{aligned}
$$

For arbitrary $f, g \in H, z \in \mathbb{R}_{e}$, we may write:

$$
\begin{gathered}
\left(\mathcal{R}_{z}\left(G^{-1}\right) f, g\right)=\left(J \mathcal{R}_{z}^{*}(G) J f, g\right)=\left(\mathcal{R}_{z}(G) J g, J f\right) \\
=\int \frac{1}{s-z} d\left(E_{G}(s) J g, J f\right)=\int \frac{1}{s-z} d(E(s) f, g)=\left(\mathcal{R}_{z}(T) f, g\right) .
\end{gathered}
$$

Therefore $T=G^{-1}$. Notice that

$\mathcal{R}_{z}(J|A| J)=\left(J|A| J-z E_{H}\right)^{-1}=\left(J\left(|A|-\bar{z} E_{H}\right) J\right)^{-1}=J \mathcal{R}_{z}^{*}(|A|) J, \quad z \in \mathbb{R}_{e}$.

For arbitrary $f, g \in H, z \in \mathbb{R}_{e}$, we may write:

$$
\begin{aligned}
\left(\mathcal{R}_{z}(J|A| J) f, g\right) & =\left(J \mathcal{R}_{z}^{*}(|A|) J f, g\right)=\left(\mathcal{R}_{z}(|A|) J g, J f\right) \\
=\int \frac{1}{\sqrt{s}-z} d\left(E_{G} J g, J f\right) & =\int \frac{1}{\sqrt{s}-z} d(E f, g)=\left(\int \frac{1}{\sqrt{s}-z} d E f, g\right) \\
& =\left(\mathcal{R}_{z}\left(\sqrt{G^{-1}}\right) f, g\right) .
\end{aligned}
$$

Therefore

$$
J|A| J=\sqrt{G^{-1}} .
$$

Let us check that

$$
\sqrt{G^{-1}}=(\sqrt{G})^{-1}
$$

In fact, using the change of variable:

$$
\lambda=\pi(u)=\left\{\begin{array}{cc}
\sqrt{u}, & u \geq 0 \\
u, & u<0
\end{array},\right.
$$

for the spectral measure $E_{G}$ (see, e.g., [1]) we obtain the spectral measure $E_{\sqrt{G}}$ of $\sqrt{G}$, and we may write:

$$
\begin{aligned}
(\sqrt{G})^{-1} & =\left(\int \sqrt{u} d E_{G}\right)^{-1}=\left(\int \lambda d E_{\sqrt{G}}\right)^{-1} \\
& =\int \frac{1}{\lambda} d E_{\sqrt{G}}=\int \frac{1}{\sqrt{u}} d E_{G}(u) .
\end{aligned}
$$


On the other hand, using the change of variable:

$$
\lambda=\widehat{\pi}(s)=\left\{\begin{array}{ll}
\frac{1}{s}, & s>0 \\
s, & s \leq 0
\end{array},\right.
$$

for the spectral measure $E$ of $G^{-1}$, we obtain the spectral measure $E_{G}$, and we may write

$$
\sqrt{G^{-1}}=\int \sqrt{s} d E(s)=\int \frac{1}{\sqrt{\lambda}} d E_{G}(\lambda) .
$$

By (11),(12) we conclude that relation (10) holds.

By (9),(10) we obtain that $J|A| J=|A|^{-1}$. Thus, $B:=|A|$ is $J$-unitary.

Consider the polar decomposition for $A$ : $A=U B$, where $U$ is a unitary operator in $H$ (since $\overline{R(A)}=\overline{R(B)}=H$ ). Then $A^{*}=B^{*} U^{*}$ (since $U$ is bounded on $H$ ) and

$$
\begin{aligned}
U B^{-1}=\left(B U^{-1}\right)^{-1} & =\left(A^{*}\right)^{-1}=J A J=J U J J B J \\
& =J U J B^{-1} .
\end{aligned}
$$

Therefore

$$
U h=J U J h, \quad h \in D(B) .
$$

By the continuity of $U$ and $J$ we conclude that $U$ is $J$-real.

Let us check assertion 2) of the theorem. For the operator $A$ in this case we may write:

$$
\begin{gathered}
J A J=J U J J B J=U B^{-1}, \\
A^{-1}=B^{-1} U^{-1} .
\end{gathered}
$$

Since $U$ is bounded on $H$, we may write:

$$
J A^{*} J=(J A J)^{*}=\left(U B^{-1}\right)^{*}=\left(B^{-1}\right)^{*} U^{*}=B^{-1} U^{-1}=A^{-1} .
$$

Therefore $A$ is $J$-unitary.

Corollary 1. Let $J$ be a conjugation on a Hilbert space $H$, and $A$ be a $J$-unitary operator in $H$. Then operators $A^{*} A$ and $A A^{*}$ are unitarily equivalent.

Proof. In the notations of Theorem 2.1 we may write: $A^{*} A=B^{2}$, and, since $U$ is bounded, $A A^{*}=U B(U B)^{*}=U B B^{*} U^{*}=U B B U^{-1}=U A^{*} A U^{-1}$.

As it was noticed in [3], for the unilateral shift $A$ the operators $A^{*} A$ and $A A^{*}$ are not unitarily equivalent. Thus, the unilateral shift is not $J$-unitary.

Example 1. (An unbounded $J$-unitary operator) Let

$$
A_{0}:=A_{0}(\beta):=\left(\begin{array}{cc}
0 & \beta i \\
-\beta i & 0
\end{array}\right) \quad \beta \in(-1,1) .
$$


Observe that

$$
\left(A_{0}(\beta) \pm I_{2}\right)^{-1}=\frac{1}{1-\beta^{2}}\left(\begin{array}{cc} 
\pm 1 & -\beta i \\
\beta i & \pm 1
\end{array}\right)
$$

Let $H=\bigoplus_{k=1}^{\infty} H_{k}$, where $H_{k}=\mathbb{C}^{2}$ is the space of 2-dimensional complex vectors, and $A=\bigoplus_{k=1}^{\infty} A_{0}\left(1-\frac{1}{k}\right)$. For an element of $H$ of the form $h=$ $\left(h_{j}\right)_{j=1}^{\infty}, h_{j}=\left(\begin{array}{c}h_{j, 1} \\ h_{j, 2}\end{array}\right) \in H_{j}$, we set $J h=\left(\mathcal{J} h_{j}\right)_{j=1}^{\infty}$, where $\mathcal{J} h_{j}=\left(\overline{\frac{h_{j, 1}}{h_{j, 2}}}\right)$. Observe that $J$ is a conjugation on $H$. It is straightforward to check that $A$ is a bounded self-adjoint, $J$-skew-self-adjoint operator on $H$, and there exist $\left(E_{H} \pm A\right)^{-1}$. Let $e_{k, 1}$ be an element of $H$ of the form $\left(h_{j}\right)_{j=1}^{\infty}, h_{j} \in H_{j}$, where $h_{j}=\delta_{j, k}\left(\begin{array}{c}1 \\ 0\end{array}\right) ; k \in \mathbb{N}$. Observe that

Since

$$
\left(E_{H}+A\right)^{-1} e_{k, 1}=\left(\delta_{j, k} \frac{1}{1-\left(1-\frac{1}{k}\right)^{2}}\left(\begin{array}{c}
1 \\
\left(1-\frac{1}{k}\right) i
\end{array}\right)\right)_{j=1}^{\infty} .
$$

$$
\left(\left(E_{H}+A\right)^{-1} e_{k, 1}, e_{k, 1}\right)_{H}=\frac{1}{1-\left(1-\frac{1}{k}\right)^{2}} \rightarrow \infty
$$

as $k \rightarrow \infty$, then $\left(E_{H}+A\right)^{-1}$ is unbounded. Consider the following operator:

$$
V=\left(A+E_{H}\right)\left(A-E_{H}\right)^{-1}=E_{H}+2\left(A-E_{H}\right)^{-1} .
$$

Transformation (15), which connects some $J$-skew-symmetric and $J$-isometric operators, was studied by Kamerina in [5]. Observe that

$$
V=\int \frac{\lambda+1}{\lambda-1} d E_{A}(\lambda)
$$

where $E_{A}(\lambda)$ is the spectral measure of $A$. Thus, $V$ is self-adjoint, and we may write:

$$
\begin{aligned}
J V^{*} J=J V J & =E_{H}+2 J\left(A-E_{H}\right)^{-1} J=E_{H}-2\left(A+E_{H}\right)^{-1} \\
& =\left(A-E_{H}\right)\left(A+E_{H}\right)^{-1}=V^{-1} .
\end{aligned}
$$

Thus, $V$ is a $J$-unitary operator. Therefore $V^{-1}$ is an unbounded $J$-unitary operator.

Unitary $J$-real operators, which appear in the refined polar decomposition (8), also play an important role in the question of an extension of $J$-imaginary symmetric operators to $J$-imaginary self-adjoint operators.

Theorem 2.2. Let $J$ be a conjugation on a Hilbert space $H$. Let $A$ be a closed J-imaginary symmetric operator in $H, \overline{D(A)}=H$. Then there exists a J-imaginary self-adjoint operator $\widetilde{A} \supseteq A$ in a Hilbert space $\widetilde{H} \supseteq H$ (with an extension of $J$ ). If the defect numbers of $A$ are equal, then there exists a $J$-imaginary self-adjoint operator $\widehat{A} \supseteq A$ in $H$. 
Proof. First, suppose that the defect numbers of $A$ are equal. Consider Cayley's transformation of $A$ :

$U_{z}=U_{z}(A)=\left(A-\bar{z} E_{H}\right)\left(A-z E_{H}\right)^{-1}=E_{H}+(z-\bar{z})\left(A-z E_{H}\right)^{-1}, \quad z \in \mathbb{C}$.

Observe that

$$
J \mathcal{M}_{z}(A)=\mathcal{M}_{-\bar{z}}(A), \quad z \in \mathbb{C},
$$

where $\mathcal{M}_{\lambda}(A):=\left(A-\lambda E_{H}\right) D(A), \lambda \in \mathbb{C}$. In particular, we see that

$$
J \mathcal{M}_{ \pm i}(A)=\mathcal{M}_{ \pm i}(A) .
$$

Then

$$
J \mathcal{N}_{ \pm i}(A)=\mathcal{N}_{ \pm i}(A)
$$

where $\mathcal{N}_{\lambda}(A):=H \ominus \mathcal{M}_{\lambda}(A), \lambda \in \mathbb{C}$.

Let $W$ be an arbitrary linear $J$-real isometric operator, which maps $\mathcal{N}_{i}(A)$ onto $\mathcal{N}_{-i}(A)$. In particular, if $\mathfrak{A}_{ \pm}=\left\{f_{k}^{ \pm}\right\}_{k=0}^{\tau}, 0 \leq \tau \leq+\infty$, is an orthonormal basis in $\mathcal{N}_{ \pm i}(A)$, corresponding to $J$ (i.e. $J f_{k}^{ \pm}=f_{k}^{ \pm}$), then we may set

$$
W \sum_{k=0}^{\tau} \alpha_{k} f_{k}^{+}=\sum_{k=0}^{\tau} \alpha_{k} f_{k}^{-}, \quad \alpha_{k} \in \mathbb{C} .
$$

Then $V:=U_{i} \oplus W$ is a $J$-real unitary operator in $H$. Observe that $\widetilde{A}:=$ $i E_{H}+2 i\left(V-E_{H}\right)^{-1} \supseteq A$, is self-adjoint and $J$-imaginary.

In the case of unequal defect numbers, we may consider an operator $\mathcal{A}:=$ $A \oplus(-A)$ in a Hilbert space $\mathcal{H}:=H \oplus H$ with a conjugation $\mathcal{J}=J \oplus J$. The operator $\mathcal{A}$ is closed symmetric, $\mathcal{J}$-imaginary, $\overline{D(\mathcal{A})}=\mathcal{H}$, and it has equal defect numbers. Thus, we may apply to $\mathcal{A}$ the already proved part.

Example 2. (A $J$-imaginary symmetric operator) Consider the usual space $H=l_{2}$ of square summable sequences of complex numbers

$$
h=\left(\begin{array}{c}
h_{0} \\
h_{1} \\
h_{2} \\
\vdots
\end{array}\right) .
$$

A conjugation $J$ will be the following one:

$$
J h=\left(\begin{array}{c}
\overline{h_{0}} \\
\overline{h_{1}} \\
\overline{h_{2}} \\
\vdots
\end{array}\right) .
$$


An operator $A$ we shall define on a set of all finite vectors $\mathcal{F}$ (i.e. vectors which components are zeros except for a finite number) by the following matrix multiplication:

$$
A h=i\left(\begin{array}{ccccc}
0 & \alpha_{0} & 0 & 0 & \ldots \\
-\alpha_{0} & 0 & \alpha_{1} & 0 & \ldots \\
0 & -\alpha_{1} & 0 & \alpha_{2} & \ldots \\
\vdots & \vdots & \vdots & \vdots & \ddots
\end{array}\right) h .
$$

It is straightforward to check that $A$ is symmetric and $J$-imaginary. Observe that $\bar{A}$ is $J$-imaginary, as well. Applying Theorem 2.2 to the operator $\bar{A}$ we conclude that the operator $A$ has a self-adjoint $J$-imaginary extension in a Hilbert space $\widetilde{H} \supseteq H$.

Acknowledgment. The author is grateful to the referee for useful comments and suggestions.

\section{REFERENCES}

[1] M. Sh. Birman and M. Z. Solomyak, Spectral Theory of Self-adjoint Operators in a Hilbert Space, Izdat. Leningradskogo univ., Leningrad, 1980.

[2] S. R. Garcia and M. Putinar, Complex symmetric operators and applications, Trans. Amer. Math. Soc., 358 (2006), 1285-1315.

[3] S. R. Garcia and M. Putinar, Complex symmetric operators and applications II, Trans. Amer. Math. Soc., 359 (2007), 3913-3931.

[4] S. R. Garcia, E. Prodan and M. Putinar, Mathematical and physical aspects of complex symmetric operators, J. Phys. A, Math. Theor, 47 (2014), 353001 (54pp).

[5] T. B. Kalinina, On extensions of an operator in a Hilbert space with an anti-unitary transformation, (Russian), Funkts. Anal., Ulyanovsk. Gos. Ped. Inst., Ulyanovsk, 17 (1981), 68-75.

[6] C. G. Li and T. T. Zhou, Skew symmetry of a class of operators, Banach J. Math. Anal., 8 (1) (2014), 279-294.

[7] I. V. Shelepov, Models of nonself-adjoint operators, commuting and anti-commuting with an involution, and an invariant factorization of their characteristic operator functions, (Russian), Abstract for a Ph.D. Thesis, Donetsk, 1992.

[8] M. H. Stone, Linear transformations in Hilbert space and their applications to analysis, AMS Colloquium Publications, Vol. 15, Providence, Rhode Island, 1932.

[9] S. M. Zagorodnyuk, On a J-polar decomposition of a bounded operator and matrices of $J$-symmetric and J-skew-symmetric operators, Banach J. Math. Anal., 4 (2) (2010), $11-36$.

(Received: September 10, 2014)

(Revised: December 6, 2014)
School of Mathematics and Mechanics Karazin Kharkiv National University Kharkiv 61022

Ukraine

Sergey.M.Zagorodnyuk@univer.kharkov.ua 